\title{
Size, weight and size of first maturation of the sea cucumber Isostichopus badionotus north of Pinar del Río, Cuba
}

\author{
Aracely Hernández-Betancourt ${ }^{1}$, Amanda Trujillo Valdés ${ }^{2}$, Patricia Canet $^{1}$, Rafael \\ Palma ${ }^{1}$ \\ ${ }^{1}$ Centro de Investigaciones Pesqueras, Cuba \\ ${ }^{2}$ Facultad de Biología, Universidad de La Habana, Cuba
}

\begin{abstract}
:
The measurement of size and weight are base variables that are used directly in the determination of indicators for the management and conservation of fish stocks. Determining the size and weight of first maturity allows us not only to define the legal minimum size and weight, but also to properly calculate the adult density, the fishable biomass and with this the maximum allowable catch or catch quota. The present research aims to determine the sizes, weights and the size of first sexual maturity of the sea cucumber I. badionotus in Arroyos de Mantua, Pinar del Río, Cuba. Size and weight data were collected at the fishing sites through independent samplings of the fishery between 2017 and 2019 during the closed season. Variable differences between fishing sites and between years were analyzed using the nonparametric Kruskal-Wallis analysis of variance. Height and weight at first sexual maturity (L50 and Pe50, respectively) were obtained by applying the cumulative distribution function. The sizes tend to increase in time and spatially as they move away from the port, without
\end{abstract}


showing statistically significant differences between years and between sites. An $\mathrm{L}_{50}=19 \mathrm{~cm}$ of total ventral length and $\mathrm{Pe}_{50}=198 \mathrm{~g}$ of wet gutted weight were obtained. The length-weight relationship turned out to be weak and positive $\left(\mathrm{R}^{2}=0.38\right)$. The management strategy of the resource in this region should include the rotation of areas in the captures and establish the L50 and Pe50 obtained as minimum capture values.

Keywords: holothuroid, sexual maturation, legal minimum size

\section{Introducción}

La medida de talla y peso son variables base que se emplean directamente en la determinación de indicadores para el manejo y conservación de stock pesqueros (Purcell 2010). Estas permiten definir valores límites de tamaño para proteger a los juveniles permitiéndoles una o más temporadas de reproducción antes de ser capturados (Purcell, Gossuin y Agudo, 2009ª en Purcell 2010). Determinar la longitud de primera maduración nos permite no solo definir la talla mínima legal, si no también, calcular adecuadamente la densidad de adultos, la biomasa pescable y con esto la captura máxima permisible o cuota de captura. Estas variables son ampliamente utilizadas en el ordenamiento y manejo de pesquerías de pepino de mar (ToralGranda 2008, Purcell 2010). Los límites de tamaños para los pepinos de mar generalmente se han basado en el tamaño de primera madurez sexual (Bruckner 2006). Usualmente la talla de primera madurez se expresa como la longitud total a la cual el $50 \%$ de los individuos están maduros (L L $_{50}$ estimada típicamente por un modelo logístico (Hamel et al. 1993, Guzmán et al. 2003). La talla mínima o de primera reproducción se basa en el tamaño en el cual el pepino alcanza la madurez sexual para asegurar que la población se reproduzca al menos una vez antes de entrar en la pesquería. Algunas regiones toman en cuenta dichas medidas precautorias para lograr una mayor conservación de las especies que son sometidas a explotación pesquera (Ortega Martínez 2015). El valor de esta medida es casi siempre menor a la mitad de las tallas máximas observadas en aquellas poblaciones que no están sujetas a aprovechamiento pesquero. Se corresponde aproximadamente a un peso menor al $30 \%$ de los pesos máximos observados (Zetina et al. 2002).

Al norte de Pinar del Río, Cuba, se realizan capturas de pepino de mar Isostichopus badionotus desde 2013 por parte de la flota pepinera perteneciente a la Unidad Económica de Base (UEB) de Arroyos de Mantua de la empresa pesquera estatal EPICOL. Desde el 
comienzo de la pesquería se ha determinado la cuota de captura basada en un porcentaje de la biomasa pescable de adultos. Como medida precautoria se impuso una talla mínima legal de $19 \mathrm{~cm}$ de largo total ventral, sin estudio directo del stock, y se definieron como adultos aquellos ejemplares con talla mayor o igual a este valor. La presente investigación tiene como objetivos determinar las tallas, pesos y la talla y el peso de primera madurez sexual del pepino de mar I. badionotus en Arroyos de Mantua, Pinar del Río, Cuba.

\section{Materiales y Métodos}

\section{Descripción del área de estudio}

Al norte de Pinar del Río, Cuba, existen cuatro sitios de pesca de pepino de mar I. badionotus que suman un área de $59 \mathrm{~km}^{2}$ (Fig. 1). La profundidad media donde se realizan las operaciones de pesca de pepino de mar es de $3 \mathrm{~m}$, el fondo es areno-fangoso, con parches de fanerógamas.

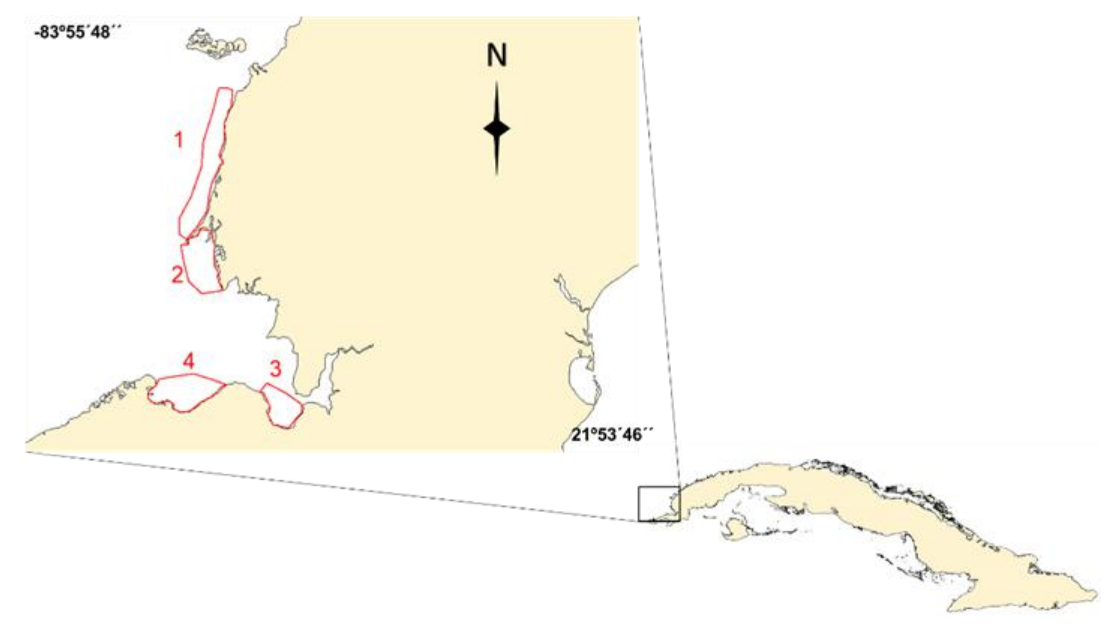

Figura 1. Sitios de pesca del pepino de mar I. badionotus en Arroyos de Mantua, al noroeste de Pinar del Río, Cuba.

\section{Procedimiento de colecta y mediciones de longitud y peso de los pepinos de mar}

Se realizaron muestreos biológicos independientes de la pesquería en los años 2017, 2018 y 2019, durante los meses de veda reproductiva (junio-octubre) en los cuatro sitios de pesca. Los datos se tomaron de los ejemplares capturados en transectos al azar, de $400 \mathrm{~m}^{2}$ de área, cada uno. Los pepinos de mar capturados fueron depositados en un estanque con agua de mar en la embarcación durante al menos 10 min para evitar ruptura de la pared corporal y evisceración. Posteriormente se determinó la longitud ventral (LT) de cada ejemplar, con una cinta métrica de $0.1 \mathrm{~cm}$ de precisión. Cada pepino de mar se abrió por la región ventral y se le 
extrajo todo el contenido interior quedando solo el tegumento o pared corporal. Luego se procedió al pesaje con una balanza analítica de precisión $0,1 \mathrm{~g}$ y se registraron todos los pesos húmedos eviscerados $(\mathrm{Pe})$. Se definieron como ejemplares maduros sexualmente aquellos que presentaban las gónadas diferenciadas.

\section{$\underline{\text { Análisis estadístico }}$}

Para obtener $\mathrm{L}_{50}$ y $\mathrm{Pe}_{50}$ (talla y peso eviscerado de primera maduración) en la zona de estudio se aplicó la función de distribución acumulativa en el programa Minitab 17. La relación tallapeso se determinó mediante una correlación de Spearman. Las comparaciones de tallas medias y de Pe medios entre años y zonas se realizaron mediante el análisis de varianza no paramétrico Kruskal-Wallis en el programa Minitab 17.

Para todas las pruebas de hipótesis estadísticas que se realizaron con los datos del estudio se prefijó un nivel de significación del $5 \%$ y se consideró el resultado significativo cuando la probabilidad asociada al estadígrafo en cuestión (p-valor) resultó menor que el nivel de significación prefijado.

\section{$\underline{\text { Resultados }}$}

Se midieron un total de 744 individuos de pepino de mar I. badionotus con tallas entre 10 y 26 $\mathrm{cm}$ de LT y una media total de $19 \mathrm{~cm}(\mathrm{DE} \pm 2,63)$. El peso eviscerado húmedo (Pe) varió entre 45 y $380 \mathrm{~g}$, con una media de $189.15 \mathrm{~g}(\mathrm{DE} \pm 47.7)$. La talla y el peso al cual el $50 \%$ del stock presentó madurez sexual ( $\mathrm{L}_{50}$ y $\mathrm{Pe}_{50}$, respectivamente) fue de $19.00 \mathrm{~cm}$ de largo total ventral y $197.5 \mathrm{~g}$ de peso eviscerado húmedo (Fig. 2). El $52 \%$ del total de pepinos medidos presentaron tallas mayores a $\mathrm{L}_{50}(19 \mathrm{~cm})$.
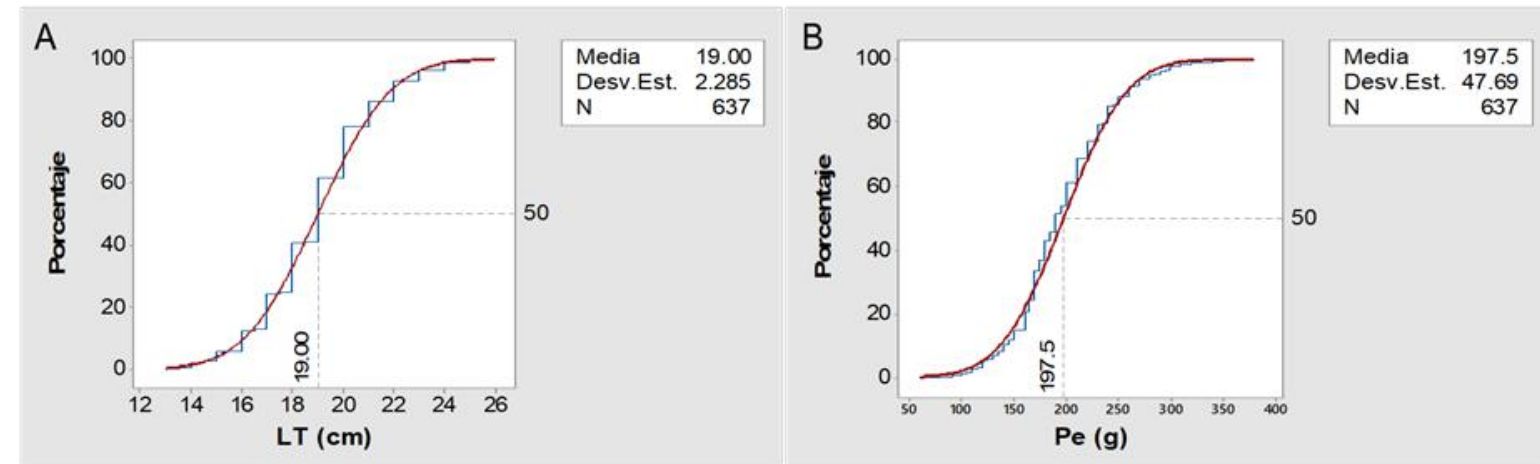

Figura 2. Función de distribución acumulativa de A: largo total ventral (LT), B: peso eviscerado húmedo (Pe), del pepino de mar I. badionotus en el periodo de veda de los años 2017, 2018 y 2019 en Pinar del Río, Cuba. 
Las tallas y los pesos medios mostraron un aumento en el tiempo, sin embargo, no existen diferencias significativas de las tallas y los pesos entre los años de estudio (p>0.05) (Fig. 3). Las tallas y los pesos no mostraron diferencias significativas entre sitios $(\mathrm{p}>0.05)$.

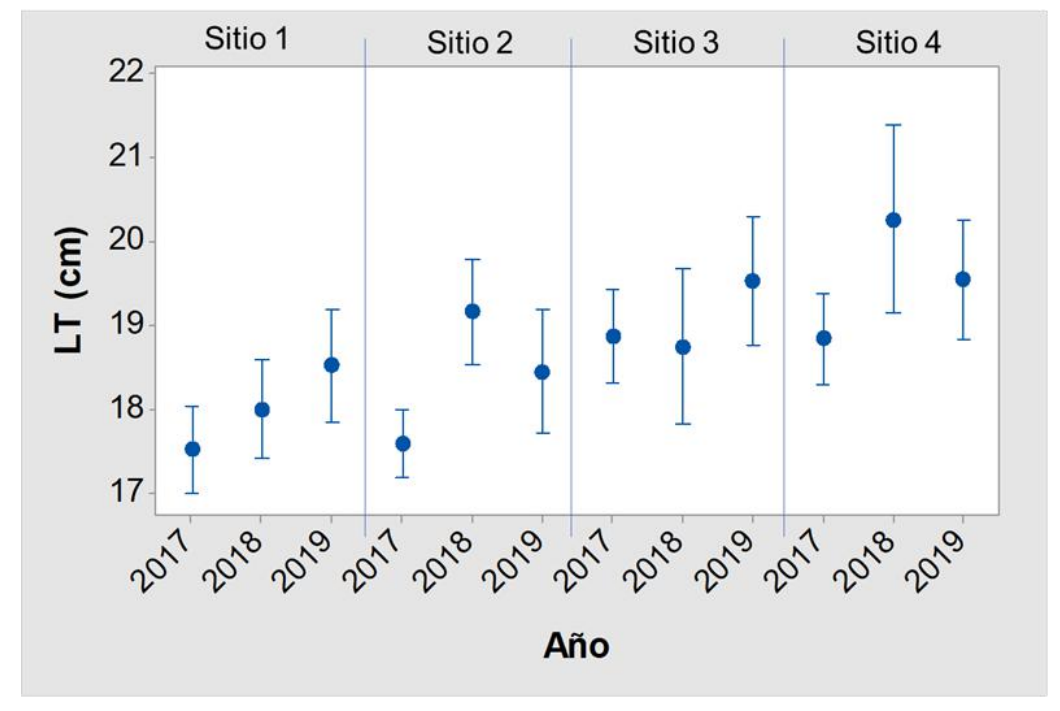

Figura 3. Largo total ventral medio (LT) del pepino de mar I. badionotus en cada sitio de pesca durante los años de estudio, en Pinar del Río, Cuba.

Las mayores tallas se observaron en los sitios $4(\mathrm{~N}=169)$ y $3(\mathrm{~N}=181)$ con valores medios de $19.24 \mathrm{~cm}$ y $18.99 \mathrm{~cm}$, respectivamente. En el sitio $2(\mathrm{~N}=226)$ se obtuvo una media de 17.99 cm de LT, mientras que en el sitio $1(\mathrm{~N}=168)$ la media fue de $17.83 \mathrm{~cm}$ de LT.

Los mayores pesos medios se observaron en los sitios $3(\mathrm{~N}=181)$ y $4(\mathrm{~N}=169)$ con valores medios de 198.62 g y 194.38 g, respectivamente (Fig. 4). Los sitios 1 (N=168) y 2 (N=226) arrojaron medias de 188.03 y $178.52 \mathrm{~g}$, respectivamente.

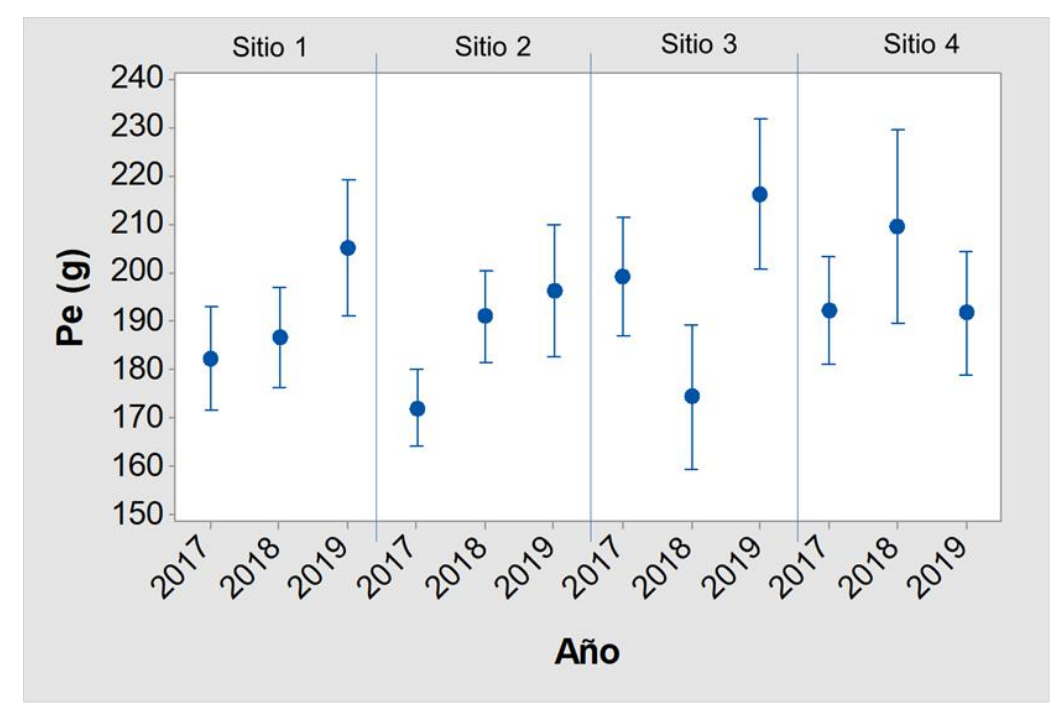

Figura 4. Peso eviscerado húmedo medio (Pe) del pepino de mar I. badionotus en cada sitio de pesca durante los años de estudio, en Pinar del Río, Cuba. 
Los mayores porcentajes de ejemplares con tallas inferiores a $\mathrm{L}_{50}$ y $\mathrm{Pe}_{50}$ se encontraron en los sitios 1 y 2 (Fig.5). De manera general existe correlación débil y positiva entre la talla y el peso. No se evidencia una relación monotónica entre dichas variables, presentando un coeficiente de correlación de Spearman $r_{s}=0.03$ y un valor $p=0.48$ (Fig. 6).

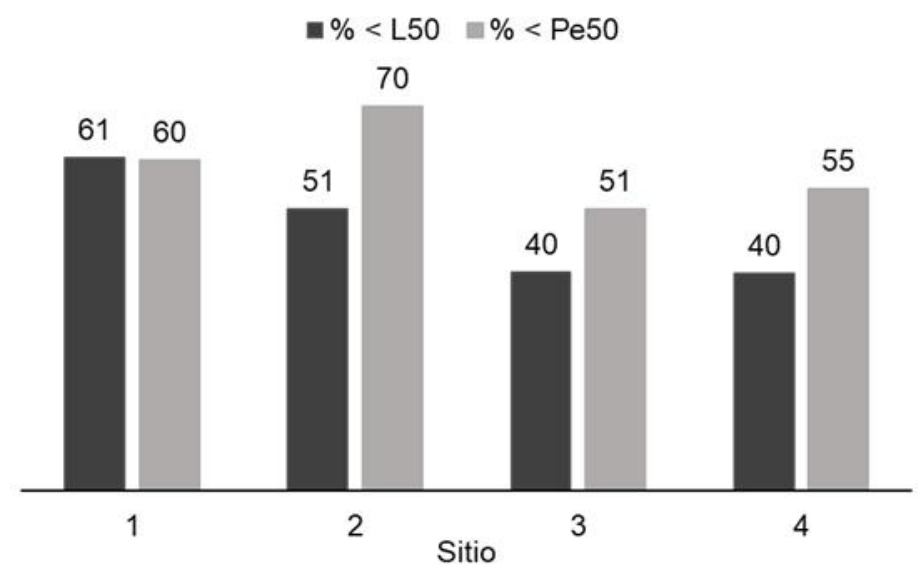

Figura 5. Porcentaje de ejemplares con tallas y pesos menores a la talla de primera maduración ( $\left.L_{50}\right)$ y peso de primera maduración ( $\mathrm{Pe}_{50}$ ), respectivamente, en cada sitio de pesca del pepino de mar $I$. badionotus durante los años 2017, 2018 y 2019, en Pinar del Río, Cuba.

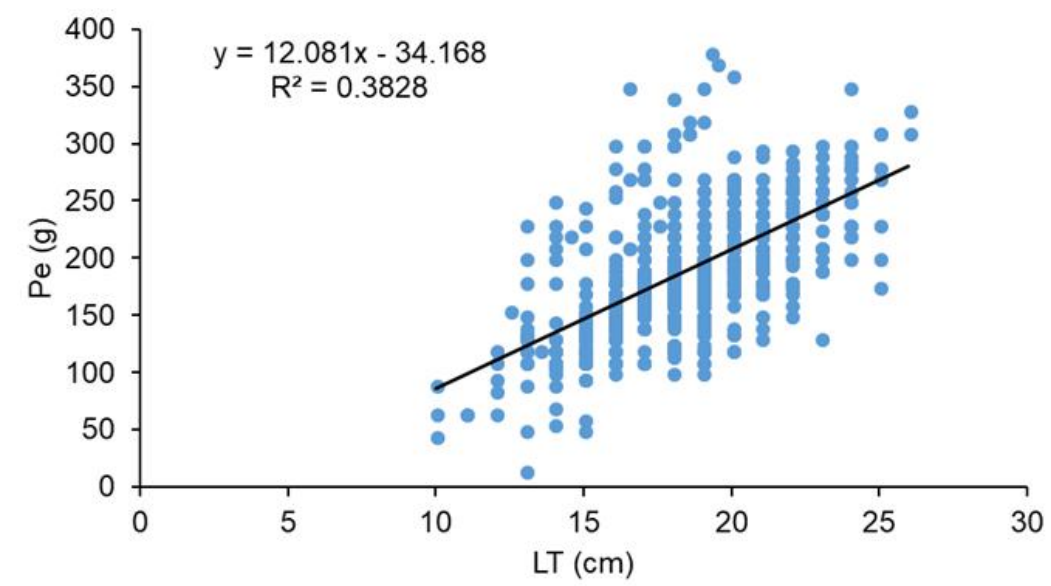

Figura 6. Relación talla-peso del pepino de mar I. badionotus para los años 2017, 2018 y 2019 en Pinar del Río, Cuba.

\section{Discusión}

\section{Talla y peso}

Las tallas registradas en el presente estudio (10-26 cm de LT) se encuentran en el rango de tallas reportadas para la especie en otras regiones del Caribe y del mundo en general. Sin embargo, esta especie es más pequeña al Norte de Pinar del Río, en relación con otros 
reportes, ya que la talla máxima y media representan aproximadamente la mitad de los valores reportados en otras regiones. En el Caribe panameño se reportó un rango de tallas de $I$. badionotus entre 16 y $41 \mathrm{~cm}$ de largo total, con un promedio de $27.05 \mathrm{~cm}$ (Guzmán et al. 2003). Mientras que, en la Península de Yucatán, México se reportó un rango entre 3 y $45 \mathrm{~cm}$ de largo total dorsal con un valor medio de $26.8 \mathrm{~cm}$, que representa aproximadamente $2 \mathrm{~cm}$ superior al largo total ventral, por lo que se puede inferir que el rango fue entre 1 y $43 \mathrm{~cm} \mathrm{y}$ una media de $24.8 \mathrm{~cm}$ de largo total ventral (Poot-Salazar 2015). En la región suroriental de Cuba se han reportado rangos de tallas entre 8-46 cm y 19.8-24.5 cm (Frías et al. 2008; Hernández-Betancourt et al. 2018), con valores medios de $21 \mathrm{~cm}$ de longitud ventral. En la Isla de la Juventud, en la región suroccidental de Cuba, las tallas reportadas presentan valores semejantes a las obtenidas en este estudio con un rango entre 14 y $30 \mathrm{~cm}$ de largo total ventral (Ortega 2015). Esta última región, es la más cercana geográficamente en relación con otras donde se estudia esta especie. Esto puede indicar que, al occidente de Cuba, tanto al norte como al sur, el pepino de mar I. badionotus adulto presenta tallas pequeñas.

Los valores de peso húmedo eviscerado (45-380 g, media de $189.15 \mathrm{~g}$ ) muestran la misma conclusión al obtenerse valores máximos y medios menores que en las regiones mencionadas. En Panamá se reportó un rango de 82-426 g con un promedio de 224 g (Guzmán et al. 2003). En México se reportó un rango de 1-800g con un promedio de 552 g (Poot-Salazar 2015). En la región suroriental de Cuba se reportaron rangos de 214.6-406 g y 50-1030 g, con un promedio de 425 g (Frías et al. 2008; Hernández-Betancourt et al. 2018). Ortega (2015) al norte de la Isla de la Juventud reporta valores que van desde los $100 \mathrm{~g}$ hasta más de $500 \mathrm{~g}$.

Los valores de peso se corresponden con el morfotipo III descrito por Vergara et al., (2018) que presentó un peso medio de 192.9 g. Los morfotipos I y II descritos por dichos autores, presentaron valores medios de 232.4 y 470.4 g, respectivamente. Los ejemplares de Pinar del Río pueden pertenecer al morfotipo III considerado una especie diferente y denominada por estos autores como Isostichopus sp. Según Acosta et al., (2021) Isostichopus sp. presenta menores tallas que I. badionotus. Estos autores obtuvieron tallas medias de $19.3 \mathrm{~cm}$ de largo total en Isostichopus sp. y $32.4 \mathrm{~cm}$ de largo total en I. badionotus. Esto puede justificar los tamaños pequeños de los ejemplares identificados como I. badionotus en Pinar del Río, en relación con esta especie en otras regiones de Cuba, cuando en realidad son especies diferentes.

Las tallas presentaron una tendencia a aumentar tanto espacial, como temporalmente. Los sitios de estudio están enumerados en orden ascendente según se alejan de puerto, de forma 
que se encontraron las tallas menores en los sitios más cercanos a puerto (1 y 2) y las tallas mayores en los sitios más alejados (3 y 4). En cada sitio, la tendencia de las tallas medias entre el primer y el último año de estudio fue ascendente. Con los valores medios de Pe ocurre lo mismo, excepto en el sitio 4, donde se mantiene una estabilidad. Aun cuando estadísticamente estos valores no difieren entre años, ni entre sitios, biológicamente puede estar sucediendo un proceso que se puede volver más marcado en los siguientes años. El hecho de que las tallas y pesos menores, así como los mayores porcentajes de juveniles se encuentran en los sitios más cercanos a puerto, puede estar indicando una sobre pesca de adultos y un reclutamiento proveniente de los sitios más alejados de puerto. Esta es la conclusión más probable, teniendo en cuenta que el estudio se realizó en el mismo rango de profundidad en todos los sitios. Esta diferencia de tallas entre sitios debe tenerse en cuenta para futuras investigaciones y en el manejo de la pesquería de este preciado recurso.

\section{Relación longitud-peso}

El bajo coeficiente de determinación $\left(\mathrm{R}^{2}=0.38\right)$ obtenido demuestra la débil relación que existe entre el incremento en longitud y aumento en peso en los pepinos de mar. Resultados mayores a los de este trabajo han sido descritos por López-Rocha (2012) para las poblaciones de $I$. badionotus en la costa de Yucatán $\left(\mathrm{R}^{2}=0,70\right)$, Frías et al. (2008) para la región suroriental $\left(\mathrm{R}^{2}=0,78\right)$ y por Poot-Salazar $(2014)$ también en Yucatán $\left(\mathrm{R}^{2}=0.52\right)$. HernándezBetancourt (2016) en la región suroriental de Cuba y Guzmán y Guevara (2002) en Panamá también obtuvieron una relación débil $\left(\mathrm{R}^{2}=0,46\right.$ y $\mathrm{R}^{2}=0,43$, respectivamente) para esta especie. La relación longitud-peso tiene implicaciones para la pesquería porque la pesca se realiza cuando los organismos tienen las mejores condiciones en cuanto a peso o talla. Según Ortega (2015), no necesariamente cuando se capturen individuos de tallas mayores estos tienen también los pesos mayores.

\section{$\underline{\text { Talla y peso de primera maduración }}$}

La edad de primera maduración es un parámetro importante en el manejo de los recursos marinos y es necesario el establecimiento de una talla mínima legal de captura que proteja a los organismos hasta que maduren lo suficiente para reproducirse. Este parámetro puede ser utilizado como punto de referencia para el manejo del recurso y como estrategia de decisión para el establecimiento de una época de veda y así contribuir a la abundancia de los stocks futuros (Fajardo-León et al., 2008; Hernández-Flores et al., 2012). 
Históricamente en la zona pesquera de la UEB "Arroyos de Mantua" se ha utilizado la talla mínima legal (TML) establecida para la Isla de la Juventud, la cual equivale a $19 \mathrm{~cm}$ de longitud ventral (Resol. 338/2009). En la presente investigación se identificó una talla de primera maduración sexual $\left(\mathrm{L}_{50}\right)$ de $19 \mathrm{~cm}$ longitud ventral, la cual equivale a $21 \mathrm{~cm}$ longitud dorsal, correspondiéndose a un peso de tegumento de primera reproducción $\left(\mathrm{Pe}_{50}\right)$ de $198 \mathrm{~g}$. En el estudio realizado por Alfonso et al. (2008) para la región sur occidental del país se reportan como talla y peso de primera madurez sexual en I. badionotus, $21.5 \mathrm{~cm}$ de largo dorsal y $183 \mathrm{~g}$ de peso de tegumento. Probablemente la gran similitud entre estos resultados sea por la relativa cercanía geográfica de las dos zonas pesqueras. Lo anterior sugiere que los hábitats de preferencia por esta especie al suroccidente y al noroccidente de Pinar del Río son predominantemente estables y similares.

En la región de Yucatán, México, se determinó que el 50\% de las hembras de I. badionotus alcanzan la talla de primera reproducción a los $23.3 \mathrm{~cm}$ de longitud dorsal, con un peso de tegumento de $232 \mathrm{~g}$, equivalente a $346 \mathrm{~g}$ de peso húmedo o total (Poot-Salazar, 2010). Otros autores han reportado que esta especie en Panamá alcanza la talla de primera madurez sexual a los $15 \mathrm{~cm}$, mientras que en Venezuela a los $18 \mathrm{~cm}$ de longitud dorsal (Guzmán et al., 2003).

\section{Conclusiones}

- En la región noroccidental de Pinar del Río, Cuba, el pepino de mar I. badionotus presenta tallas y pesos pequeños en relación con los reportados para esta especie en regiones orientales del país y similares a la región suroccidental.

- Los ejemplares de menores tallas se encuentran en los sitios más cercanos a puerto, probablemente producto de una sobrepesca. Esto sugiere la aplicación de rotación de áreas en la pesca como medida de manejo del recurso en esta región.

- La talla y el peso de primera madurez sexual de $19 \mathrm{~cm}$ de LT y $198 \mathrm{~g}$ de Pe, respectivamente, obtenidas en la presente investigación, deben reportarse oficialmente cómo la talla y peso mínimo legal de la pesquería en esta región. 


\section{Bibliografía}

[1] Alfonso I., M. P. Frías., R. Castelo y Y. Blás. 2008. Situación de la pesquería del pepino de mar Isostichopus badionotus al norte de la isla de la juventud, Cuba. Rev. Cub. Invest Pesq. 25 (1): 20-26.

[2] Bruckner, A.W. 2006. Management and conservation strategies and practices for sea cucumbers. In: A.W. Bruckner (ed.) Proceedings of the CITES workshop on the conservation of sea cucumbers in the families Holothuriidae and Stichopodidae. NOAA Technical Memorandum NMFS-OPR-34, USA., pp. 74-103.

[3] Fajardo-León, M.; Suárez-Higuera M.; del Valle-Manríquez A.; Hernández-López A. 2008. Biología reproductiva del pepino de mar Parastichopus parvimensis (Echinodermata: Holothuroidea) de Isla Natividad y Bahía Tortugas, Baja California Sur, México. Revista Ciencias Marinas, 34(2): 165-177pp.

[4] Frías, M.P., I. Alfonso., R. Castelo y Y. Blás. 2008. Variaciones talla- peso del pepino de mar Isostichopus badionotus (Selenka, 1867) en las regiones suroriental y sur occidental de Cuba. Rev. Cub. Invest Pesq., 25 (1): 38-45.

[5] Guzmán H.M., C.A. Guevara. 2002. Population Structure, Distribution and Abundance of Three Commercial Species of Sea Cucumber (Echinodermata) in Panama. Caribbean Journal of Science, Vol. 38, No. 3-4, 230-238.

[6] Guzmán, H.M., C.A. Guevara., I.C. Hernández. 2003. Reproductive cycle of two commercial species of sea cucumber (Echinodermata: Holothuroidea) from Caribbean Panama. Marine Biology, 142: 271-279.

[7] Hamel, J.F., Himmelman, J.H \& L. Dufresne. 1993. Gametogenesis and spawning of the sea cucumber Psolus fabricii (Duben and Koren). Biological Bulletin. Marine Biological Laboratory, Woods Hole, 184: 125-143.

[8] Hernández Betancourt, A. 2016. Ordenamiento de la pesquería del pepino de mar (Isostichopus badionotus Selenka, 1867), al sur de Camagüey, Cuba (Tesis presentada en opción al Título Académico de Máster en Biología Marina y Acuicultura con Mención en Biología Marina). La Habana: Centro de Investigaciones Marinas Universidad de La Habana.

[9] Hernández, A., R. Palma., P. Canet. 2018. Talla, peso, densidad y potencial pesquero del pepino de mar (Isostichopus badionotus Selenka, 1867) en la región suroriental de Cuba. Centro de Investigaciones Pesqueras. 
[10] Hernández-Flores, A., P. L. Ardisson-Herrera., A. Poot-Salazar., J.C Espinoza-Méndez. 2012. Fundamento para el establecimiento de la veda de las especies de pepino de mar frente a las costas de la Península de Yucatán. Dictamen Técnico. SAGARPA. Instituto Nacional de Pesca de México. 8 pp.

[11] López-Rocha, J. A. 2012. Distribución y abundancia del pepino de mar Isostichopus badionotus frente a la Costa de Sisal, Yucatán. Proceedings of the 64th Gulf and Caribbean Fisheries Institute, 1(1), 153-160.

[12] Ortega Martínez, M. 2015. Evaluación de la pesquería del pepino de mar Isostichopus badionotus (Aspidochirotida: Sticopodidae), al norte de la Isla de la Juventud de Cuba (Tesis para obtener el grado de Maestro en Ciencias en Manejo de Recursos Marinos). La Paz, Bolivia: Instituto Politécnico Nacional Centro Interdisciplinario de Ciencias Marinas.

[13] Purcell, S.W. (2010). Manejo de las Pesquerías de Pepino de Mar con un Enfoque Ecosistémico. A. Lovatelli, M. Vasconcellos, y Y. Yimin (eds.) FAO Documento Técnico de Pesca y Acuicultura. No. 520. Roma, Italia. 169 pp.

[14] Poot-Salazar, A. 2010. Crecimiento, reproducción y hábitos alimenticios de Isostichopus badionotus Selenka, 1867 (Echinodermata: Holothuroidea) en el poniente del estado de Yucatán. Propuesta de tesis doctoral. CINVESTAV- Mérida. México.

[15] Poot-Salazar, A., Ardisson, P.-L., Poot-Salazar, E., Poot-Salazar, D.A. y Caro-Méndez, I.N. 2014. La pesca del pepino de mar en Celestún Yucatán: una búsqueda hacia el manejo sostenible. Revista Cubana de Investigaciones Pesqueras, 31: 1-4.

[16] Poot-Salazar, A. 2015. Crecimiento, reproducción y hábitos alimenticios de Isostichopus badionotus (Selenka 1867) (Echinodermata: Holothuroidea) en el poniente del estado de Yucatán. Tesis presentada para obtener el grado de Doctor en Ciencias. Centro de investigación y de estudios avanzados del Instituto Politécnico Nacional de Mérida. Departamento de recursos de mar. 153pp

[17] Resol. 338/2009, Ministerio de la Industria Pesquera Resolución. Departamento de Regulaciones Pesqueras y Ciencias del MINAL.

[18] Toral-Granda, V. (2008). "Population status, fisheries and trade of sea cucumbers in Latin America and the Caribbean"'. Pages 213-229 in: V. Toral-Granda, A. Lovatelli, and M. Vasconcellos (eds.) Sea Cucumbers. A Global Review of Fisheries and Trade. FAO Fisheries and Aquaculture Technical Paper. No. 516. Rome, Italy.

[19] Zetina, C., G. Ríos, I. Hernández, M. Guevara, E. Ortiz \& J. Pool. 2002. Catálogo de Especies de Pepinos de Mar Comercializables del Estado de Yucatán Universidad Autónoma de Yucatán, Mérida, Yucatán, México. 2002, pp 103. 